PROCEEDINGS OF THE

AMERICAN MATHEMATICAL SOCIETY

Volume 137, Number 7, July 2009, Pages 2459-2469

S 0002-9939(09)09743-3

Article electronically published on February 23, 2009

\title{
KHOVANOV-ROZANSKY HOMOLOGY AND THE BRAID INDEX OF A KNOT
}

\author{
KEIKO KAWAMURO
}

(Communicated by Daniel Ruberman)

\begin{abstract}
We construct a knot whose braid index is not detected by the Morton-Franks-Williams (MFW) inequality but is detected by a related KRMFW inequality that comes from the Khovanov-Rozansky homology. We also construct infinitely many knots whose braid indices are not detected by the KR-MFW inequality.
\end{abstract}

\section{INTRODUCTION}

Alexander's theorem states that any knot or link is isotopic to the closure of a braid 20]. We can measure the complexity of knot $\mathcal{K}$ by the minimal possible number of braid strands, which is called the braid index $b_{\mathcal{K}}$. Morton [13] and FranksWilliams [5] found an inequality which gives a lower bound for the braid index.

Let $\mathcal{K} \subset S^{3}$ be an oriented knot or link and $\mathcal{B}_{\mathcal{K}}$ be the infinite set of closed braid diagrams of $\mathcal{K}$. For an oriented closed braid diagram $D$, let $b_{D}$ denote the number of the braid strands and $w_{D}$ the writhe, which is the number of positive crossings minus the number of negative crossings of $D$. Then the braid index of $\mathcal{K}$ is $b_{\mathcal{K}}:=\min \left\{b_{D} \mid D \in \mathcal{B}_{\mathcal{K}}\right\}$.

We adopt the following definition of the HOMFLYPT polynomial $P_{\mathcal{K}}(a, q)$ defined by the skein relation:

$$
a P_{\mathcal{K}_{-}}(a, q)-a^{-1} P_{\mathcal{K}_{+}}(a, q)=\left(q-q^{-1}\right) P_{\mathcal{K}_{0}}(a, q) \text { and } P_{\text {unknot }}(a, q)=1,
$$

where $\mathcal{K}_{+}, \mathcal{K}_{-}, \mathcal{K}_{0}$ are knots or links that differ at a single site as indicated in Figure 1.

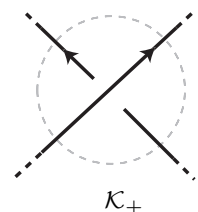

$\mathcal{K}_{+}$

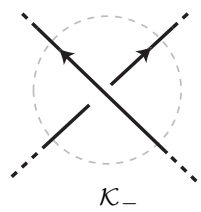

$\mathcal{K}_{-}$

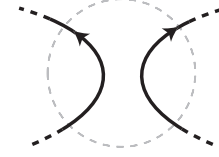

$\mathcal{K}_{0}$

FiguRE 1

Let $d_{ \pm}(\mathcal{K})$ be the maximal (resp. minimal) $a$-degree of $P_{\mathcal{K}}(a, q)$. Now we state the Morton-Franks-Williams (MFW) inequality:

Received by the editors November 9, 2007, and, in revised form, July 2, 2008.

2000 Mathematics Subject Classification. Primary 57M25, 57M27; Secondary 57M50.

The author was partially supported by NSF grants DMS-0806492 and DMS-0635607.

(C)2009 American Mathematical Society 2459 
Theorem 1.1 (Morton-Franks-Williams inequality [5, 13]). For any closed braid diagram $D \in \mathcal{B}_{\mathcal{K}}$ of knot or link $\mathcal{K}$ we have

$$
w_{D}-b_{D}+1 \leq d_{-}(\mathcal{K}) \leq d_{+}(\mathcal{K}) \leq w_{D}+b_{D}-1 .
$$

Moreover $\left(d_{+}(\mathcal{K})-d_{-}(\mathcal{K})\right) / 2+1 \leq b_{\mathcal{K}}$.

The Khovanov-Rozansky homology [10, 11] is a categorification of the HOMFLYPT polynomial. In this paper, we use the reduced HOMFLY homology $\bar{H}^{i, j, k}(\mathcal{K})$ introduced by Rasmussen [15, Definition 2.13] whose graded Euler characteristic is the HOMFLYPT polynomial: $\sum_{i, j, k}(-1)^{(k-j) / 2} a^{j} q^{i} \operatorname{dim} \bar{H}^{i, j, k}(\mathcal{K})=$ $P_{\mathcal{K}}(a, q)$.

Dunfield-Gukov-Rasmussen 3 and $\mathrm{Wu} 19$ found a Khovanov-Rozansky homology version of the MFW inequality. We call it the $K R-M F W$ inequality.

Theorem 1.2 (KR-MFW-inequality [3, 19]). Let $\mathcal{K}$ be a knot or a link and let

$$
\begin{aligned}
& \delta_{+}(\mathcal{K}):=\max \left\{j \mid \bar{H}^{i, j, k}(\mathcal{K}) \neq 0, \text { for some } i, k\right\}, \\
& \delta_{-}(\mathcal{K}):=\min \left\{j \mid \bar{H}^{i, j, k}(\mathcal{K}) \neq 0, \text { for some } i, k\right\} .
\end{aligned}
$$

Then for any closed braid diagram $D \in \mathcal{B}_{\mathcal{K}}$ of $\mathcal{K}$ we have

$w_{D}-b_{D}+1 \leq \delta_{-}(\mathcal{K}) \leq \delta_{+}(\mathcal{K}) \leq w_{D}+b_{D}-1, \quad$ i.e.,$\quad\left(\delta_{+}(\mathcal{K})-\delta_{-}(\mathcal{K})\right) / 2+1 \leq b_{\mathcal{K}}$.

Definition 1.3. The MFW (resp. KR-MFW) inequality is called sharp on $\mathcal{K}$ if there exists $D \in \mathcal{B}_{\mathcal{K}}$ such that both equalities $w_{D}-b_{D}+1=d_{-}(\mathcal{K})\left(\operatorname{resp} . \delta_{-}(\mathcal{K})\right)$ and $d_{+}(\mathcal{K})=w_{D}+b_{D}-1\left(\right.$ resp. $\left.\delta_{+}(\mathcal{K})\right)$ hold.

Since $\delta_{-}(\mathcal{K}) \leq d_{-}(\mathcal{K}) \leq d_{+}(\mathcal{K}) \leq \delta_{+}(\mathcal{K})$, we have the following.

Proposition 1.4. The sharpness of the $M F W$ (resp. KR-MFW) inequality implies

$$
\left(d_{+}(\mathcal{K})-d_{-}(\mathcal{K})\right) / 2+1=b_{\mathcal{K}} \quad\left(\operatorname{resp} .\left(\delta_{+}(\mathcal{K})-\delta_{-}(\mathcal{K})\right) / 2+1=b_{\mathcal{K}}\right) .
$$

Elrifai 4 has enumerated all the 3-braids on which the MFW inequality is nonsharp. Let $\sigma_{i}$ be the standard generator of the braid group satisfying $\sigma_{i} \sigma_{j}=\sigma_{j} \sigma_{i}$ when $|i-j|>1$ and $\sigma_{i} \sigma_{i+1} \sigma_{i}=\sigma_{i+1} \sigma_{i} \sigma_{i+1}$. For simplicity we denote $\sigma_{i}$ by $i$ and its inverse $\sigma_{i}^{-1}$ by $\bar{i}$ or $i^{-1}$.

Theorem 1.5 (Elrifai's example [4]). On all knots and links of braid index $=3$ the $M F W$ inequality is sharp except for

$$
\mathcal{K}_{k}=\left(\begin{array}{lllll}
1 & 2 & 2 & 1
\end{array}\right)^{2 k} 12^{-2 k-1} \text { and } \mathcal{L}_{k}=\left(\begin{array}{llll}
1 & 2 & 2 & 1
\end{array}\right)^{2 k+1} 12^{-2 k+1}
$$

for $k \in \mathbb{N}$ and their mirror images $\overline{\mathcal{K}_{k}}, \overline{\mathcal{L}}_{k}$.

As the Euler characteristic of the KR-homology gives the HOMFLYPT polynomial, the KR-homology contains more information than the HOMFLYPT polynomial. It is interesting to find concrete examples that show the "gap" between the KR-homology and the HOMFLYPT polynomial. Elrifai's examples seem to be natural candidates to see such a gap. In fact, we have:

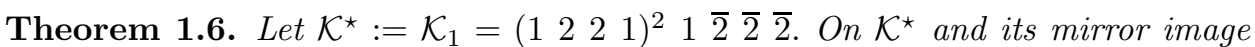
$\overline{\mathcal{K}^{\star}}$ the $M F W$-inequality is not sharp, but the KR-MFW inequality is sharp. 
These are the first examples which show that the Khovanov-Rozansky homology is "stronger" than the HOMFLYPT polynomial in terms of detecting the braid index. (Though it is natural to expect that the statement of Theorem 1.6 holds for all Elrifai's 3-braids, our proof seems to work only for a knot of small crossing number and $\mathcal{K}^{\star}$ is the simplest among them.)

However, we will also see that the Khovanov-Rozansky homology is not almighty. We study an obstruction to sharpness of the KR-MFW inequality and give infinitely many (and also first of their kind) examples of knots whose braid index is not detected by the KR-homology.

Let $B M_{x, y, z, w}$ where $x, y, z, w \in \mathbb{Z}$ be the closure of $1^{x} 2^{y} \overline{3} 2^{z} 1^{w} 23223$, where $n^{x}$ is a simplified notation for $\left(\sigma_{n}\right)^{x}$. See the sketch on the left of Figure 2 .
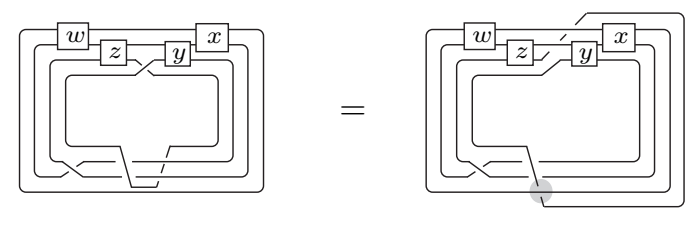

Figure 2. The BM-diagram: $1^{x} 2^{y} \overline{3} 2^{z} 1^{w} 23223$

It has been known [8] that the BM-diagram contains the five knots $9_{42}, 9_{49}, 10_{132}$, $10_{150}, 10_{156}$, on which the MFW inequality is not sharp [6]. Furthermore, it contains infinitely many four tuples $(x, y, z, w)$ where the MFW inequality is not sharp 8$]$. A parallel result holds for the KR-MFW inequality:

Theorem 1.7. There are infinitely many four tuples $(x, y, z, w)$ such that the KRMFW inequality is not sharp on $B M_{x, y, z, w}$.

Elrifai's examples have another interesting feature related to the generalized Jones conjecture [12, 8] and the maximal Bennequin number conjecture as we state below. See [6, page 357] for Jones' original conjecture.

Conjecture 1.8 (Generalized Jones conjecture). Let $\Phi: \mathcal{B}_{\mathcal{K}} \rightarrow \mathbb{N} \times \mathbb{Z}$ be a map with $\Phi(D):=\left(b_{D}, w_{D}\right)$ for $D \in \mathcal{B}_{\mathcal{K}}$. Then there exists a unique $w_{\mathcal{K}} \in \mathbb{Z}$ such that

$$
\Phi\left(\mathcal{B}_{\mathcal{K}}\right)=\left\{\left(b_{\mathcal{K}}+x+y, w_{\mathcal{K}}+x-y\right) \mid x, y \in \mathbb{N}\right\}
$$

which is a lattice in the shaded infinite region sketched in Figure 3 .

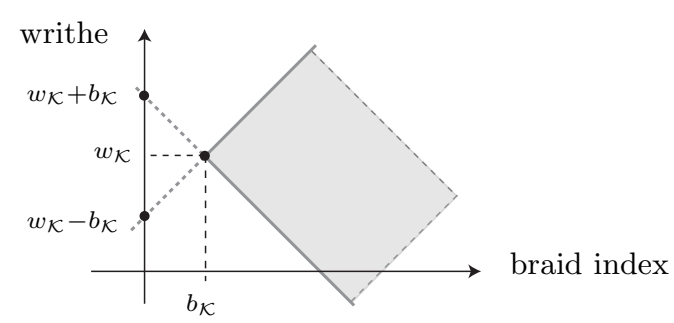

FIGURE 3. The region of braid representatives of $\mathcal{K}$

We can apply Conjecture 1.8 to contact geometry. Bennequin [1 proved that any transversal knot in the standard contact structure $\left(S^{3}, \xi_{s t d}\right)$ can be identified with 
a closed braid in $\mathbb{R}^{3}$. The Bennequin (self-linking) number $\beta_{D}:=w_{D}-b_{D}$ of the braid diagram $D \in \mathcal{B}_{\mathcal{K}}$ is an invariant of transversal knots in $\left(S^{3}, \xi_{s t d}\right)$. We denote the maximal Bennequin number of $\mathcal{K}$ by $\beta_{\mathcal{K}}:=\left\{\beta_{D} \mid D \in \mathcal{B}_{\mathcal{K}}\right\}$. Conjecture 1.8 implies the following:

Conjecture 1.9 (Maximal Bennequin number conjecture). The maximal Bennequin number $\beta_{\mathcal{K}}$ of $\mathcal{K}$ is realized at the minimal braid index and $\beta_{\mathcal{K}}=w_{\mathcal{K}}-b_{\mathcal{K}}$.

By Definition 1.3 of sharpness, it follows that:

Proposition 1.10. The sharpness of the (KR-) MFW inequality implies Conjectures 1.8 and 1.9 .

Namely, the two inequalities $w_{D}-b_{D}+1 \leq d_{-}$and $d_{+} \leq w_{D}+b_{D}-1$ in Theorem 1.1 correspond to the two boundary lines of the region in Figure 3 ,

Conjecture 1.8 holds for many classes of knots: Franks and Williams [5] proved the sharpness of the MFW inequality for knots and links that can be represented by a positive braid with a positive full twist, given by a word of the form $P \Delta^{2}$. This class includes unlinks, torus links and Lorenz links. Murasugi 14 affirmed the sharpness for alternating fibred links and 2-bridge links. Jones [6] verified the sharpness for all knots of 10 crossings or less in the standard knot table except $9_{42}, 9_{49}, 10_{132}, 10_{150}, 10_{156}$. Thus, Conjecture 1.8 holds for these knots and links by Proposition 1.10, In [9], we proved Conjecture 1.8 for $9_{42}, 9_{49}, 10_{132}, 10_{150}, 10_{156}$, by computing deficits of the MFW inequality of their cables. In [8], more general results are given: If Conjecture 1.8 holds for $\mathcal{K}, \mathcal{L}$, then Conjecture 1.8 also holds for the connect sum $\mathcal{K} \# \mathcal{L}$ and the $(p, q)$-cable of $\mathcal{K}$.

Thanks to Elrifai (Theorem 1.5) we can improve the understanding of Conjectures 1.8 and 1.9 for the set of 3-braids $B_{3}$. Let $B_{3}^{\prime}:=B_{3} \backslash\left\{\mathcal{K}_{k}, \mathcal{L}_{k}, \overline{\mathcal{K}}_{k}, \overline{\mathcal{L}}_{k} \mid k \in \mathbb{N}\right\}$. Rudolph's slice Bennequin inequality [16] will play an important role in proving:

Theorem 1.11. Conjecture 1.8 holds for $B_{3}^{\prime}, \mathcal{K}_{k}$ and $\overline{\mathcal{K}_{k}}$ for $k \in \mathbb{N}$. Conjecture 1.9 holds for $B_{3}^{\prime}, \mathcal{K}_{k}, \mathcal{L}_{k}$ and $\overline{\mathcal{K}_{k}}$ for $k \in \mathbb{N}$.

The rest of the paper is organized as follows. In Section 2, we compute the Khovanov-Rozansky homology of $\mathcal{K}^{\star}$ and prove Theorem 1.6. In Section 3, we discuss non-sharpness of the KR-MFW inequality and prove Theorem 1.7. In Section 4 we prove Theorem 1.11 .

\section{Proof of Theorem 1.6}

Throughout this section, we use the conventions and notation used in Rasmussen's paper [15].

Let $\bar{H}^{i, j, k}(\mathcal{K})$ be the reduced HOMFLY homology [15, Definition 2.13] with

$$
\sum_{i, j, k}(-1)^{(k-j) / 2} a^{j} q^{i} \operatorname{dim} \bar{H}^{i, j, k}(\mathcal{K})=P_{\mathcal{K}}(a, q),
$$

where $i$ is the $q$-grading and $j, k$ the homological gradings. Let $\bar{H}_{N}^{I, J}(\mathcal{K})$ be the reduced $\operatorname{sl}(N)$ homology of Khovanov and Rozansky [10, [15, Section 2.9] with

$$
\sum_{I, J}(-1)^{J} q^{I} \operatorname{dim} \bar{H}_{N}^{I, J}(\mathcal{K})=P_{\mathcal{K}}\left(q^{N}, q\right),
$$


where $I$ is the $q$-grading and $J$ the homological grading. Due to Rasmussen [15, Theorem 5.1], there is a spectral sequence $E_{k}(N)$ whose $E_{1}$-term is $\bar{H}(\mathcal{K})$ and which converges to $\bar{H}_{N}(\mathcal{K})$. In particular, [15, Proposition 7.3] tells us that when $\mathcal{K}$ is KR-thin (defined shortly), the spectral sequence converges at the $E_{1}$-term and the isomorphism

$$
\bar{H}_{N}^{I, J}(\mathcal{K}) \simeq \bigoplus_{\substack{i+N j=I \\(k-j) / 2=J}} \bar{H}^{i, j, k}(\mathcal{K})
$$

holds for all $N>1$. Moreover, [15, Theorem 1] proves that for any $\mathcal{K}$, the isomorphism (2.3) holds for all sufficiently large $N \gg 1$.

Let $\sigma(\mathcal{K})$ denote the signature of $\mathcal{K}$ so that positive knots have positive signatures. We introduce a new grading $\delta:=i+j+k$. A knot $\mathcal{K}$ is called $K R$-thin if $\bar{H}^{i, j, k}(\mathcal{K})=$ 0 whenever $\delta \neq \sigma(\mathcal{K})$ [15, Definition 7.2]. We say that a two-component link $\mathcal{L}$ is KR-thin if $\overline{\bar{H}}_{N}(\mathcal{L})$, the totally reduced homology of $\mathcal{L}$, is 0 whenever $\delta \neq \sigma(L)$ modulo $(2 N-4)$ for all $N \gg 1$ [15, Section 7.3]. From the definition and (2.1), when $\mathcal{K}$ is KR-thin, its homology $\bar{H}(\mathcal{K})$ is completely determined by $\sigma(\mathcal{K})$ and $P_{\mathcal{K}}(a, q)$. Rasmussen proved the following.

Theorem 2.1. (1) [15, Corollary 7.7] Let $\mathcal{K}_{+}, \mathcal{K}_{-}, \mathcal{K}_{0}$ be knots or links that differ at a single site as in Figure 1. If $\mathcal{K}_{-}, \mathcal{K}_{0}$ are KR-thin and $\operatorname{det} \mathcal{K}_{-}+2 \operatorname{det} \mathcal{K}_{0}=\operatorname{det} \mathcal{K}_{+}$, then $\mathcal{K}_{+}$is also KR-thin.

(2) [15, Corollary 7.9] The connect sum of two KR-thin knots is also KR-thin.

(3) [15. Proposition 7.10] Among the knots with less than or equal to 9 crossings, only $8_{19}, 9_{42}, 9_{43}, 9_{47}$ are not $K R$-thin.

(4) [15, Proposition 7.6] There is a long exact sequence

$$
\stackrel{(-2 N, 0)}{\longrightarrow} \bar{H}_{N}\left(\mathcal{K}_{-}\right) \stackrel{(N, 1)}{\longrightarrow} \bar{H}_{N}\left(\mathcal{K}_{0}\right) \stackrel{(N, 1)}{\longrightarrow} \bar{H}_{N}\left(\mathcal{K}_{+}\right) \stackrel{(-2 N, 0)}{\longrightarrow} \bar{H}_{N}\left(\mathcal{K}_{-}\right) \stackrel{(N, 1)}{\longrightarrow},
$$

where the pairs of numbers over the arrows indicate the degree of the corresponding map with respect to the $(q, \delta)$-bigrading.

This KR-thin property and the skein long exact sequence (2.4) will play important roles in the proof of Theorem 1.6.

Proof of Theorem 1.6. We first specify resolutions of $\mathcal{K}^{\star}$ :

$$
\begin{aligned}
\mathcal{K}^{\star}=\mathcal{K}_{+} & =122112211 \overline{2} \overline{2} \\
\mathcal{K}_{-} & =12211221 \overline{1} \overline{2} \overline{2}=12211 \overline{2}\left(=\overline{5_{2}}, \text { mirror image of } 5_{2}\right) \\
\mathcal{K}_{0} & =12211221 \overline{2} \overline{2} \\
\mathcal{K}_{0-} & =122112 \overline{2} \overline{2} \overline{2} \overline{2}=122111 \overline{2} \overline{2} \overline{2} \\
\mathcal{K}_{00} & =1221121 \overline{2} \overline{2} \overline{2}\left(=\overline{5_{2}}\right) \\
\mathcal{K}_{0--} & =12 \overline{2} 111 \overline{2} \overline{2} \overline{2}=1111 \overline{2} \overline{2} \overline{2}\left(=T_{2,-3} \# T_{2,4}\right) \\
\mathcal{K}_{0-0} & =12111 \overline{2} \overline{2} \overline{2} \text { (=unknot), }
\end{aligned}
$$

where $T_{p, q}$ is the $(p, q)$-torus knot or link. See Figure4. Thanks to Theorem 2.1. (2) and (3), the unknot, $\overline{5_{2}}$ and $T_{2,-3} \# T_{2,4}$ are KR-thin. By Theorem 2.1.(1) and

$$
\operatorname{det}\left(\mathcal{K}_{0--}\right)+2 \operatorname{det}\left(\mathcal{K}_{0-0}\right)=12+2=14=\operatorname{det}\left(\mathcal{K}_{0-}\right),
$$

link $\mathcal{K}_{0-}$ is also KR-thin. See Table 1 . Though we do not know yet whether $\mathcal{K}_{+}$ and $\mathcal{K}_{0}$ are KR-thin or not, we will see that they are actually non-thin. 


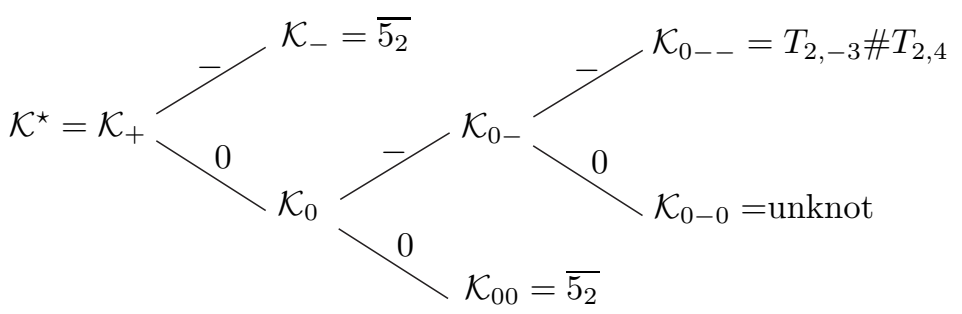

FigURE 4 . Resolutions of $\mathcal{K}^{\star}$

TABLE 1

\begin{tabular}{llcccc}
\hline & & & $\sigma$ & det & KR-thin \\
\hline $\mathcal{K}_{+}=\mathcal{K}^{\star}$ & & knot & 2 & 7 & (non-thin?) \\
\hline $\mathcal{K}_{-}=\mathcal{K}_{00}$ & $\overline{5_{2}}$ & knot & 2 & 7 & thin \\
\hline $\mathcal{K}_{0}$ & & link & 1 & 0 & (non-thin?) \\
\hline $\mathcal{K}_{0-}$ & & link & 1 & 14 & thin \\
\hline $\mathcal{K}_{0--}$ & $T_{2,-3} \# T_{2,4}$ & link & 1 & 12 & thin \\
\hline $\mathcal{K}_{0-0}$ & unknot & knot & 0 & 1 & thin \\
\hline
\end{tabular}

Let $M$ be a free module of rank $=4$ whose graded Poincaré polynomial [3] is $(q / t+t / q)^{2}$. Applying (2.4) to the triple $\left(\mathcal{K}_{+}=\mathcal{K}^{\star}, \mathcal{K}_{-}=\overline{5_{2}}, \mathcal{K}_{0}\right)$ and its analogous version (when $\mathcal{K}_{ \pm}$are links and $\mathcal{K}_{0}$ is a knot) to $\left(\mathcal{K}_{0}, \mathcal{K}_{0-}, \mathcal{K}_{00}=\overline{5_{2}}\right)$, we have (2.5) and (2.6) respectively:

$$
\begin{gathered}
\stackrel{(-2 N, 0)}{\longrightarrow} \bar{H}_{N}\left(\overline{5_{2}}\right) \stackrel{(N, 1)}{\longrightarrow} \bar{H}_{N}\left(\mathcal{K}_{0}\right) \stackrel{(N, 1)}{\longrightarrow} \bar{H}_{N}\left(\mathcal{K}^{\star}\right) \stackrel{(-2 N, 0)}{\longrightarrow} \bar{H}_{N}\left(\overline{5_{2}}\right) \stackrel{(N, 1)}{\longrightarrow}, \\
\stackrel{(-2 N, 0)}{\longrightarrow} \bar{H}_{N}\left(\mathcal{K}_{0-}\right) \stackrel{(N, 1)}{\longrightarrow} \bar{H}_{N}\left(\overline{5_{2}}\right) \otimes M \stackrel{(N, 1)}{\longrightarrow} \bar{H}_{N}\left(\mathcal{K}_{0}\right) \stackrel{(-2 N, 0)}{\longrightarrow} \bar{H}_{N}\left(\mathcal{K}_{0-}\right) \stackrel{(N, 1)}{\longrightarrow} . \\
\circ
\end{gathered}
$$

The HOMFLYPT polynomials of the resolutions of $\mathcal{K}^{\star}$ are computed by MATHEMATICA:

$$
\begin{aligned}
(2.7) \quad P_{\mathcal{K}^{\star}}= & a^{8}\left(-q^{4}-1-q^{-4}\right)+a^{6}\left(q^{6}+q^{2}+q^{-2}+q^{-6}\right) \\
(2.8) \quad P_{\overline{5_{2}}}= & -a^{6}+a^{4}\left(q^{2}-1+q^{-2}\right)+a^{2}\left(q^{2}-1+q^{-2}\right) \\
(2.9)\left(q-q^{-1}\right)^{2} P_{\overline{5_{2}}}= & a^{6}\left(-q^{2}+2-q^{-2}\right)+a^{4}\left(q^{4}-3 q^{2}+4-3 q^{-2}+q^{-4}\right) \\
& +a^{2}\left(q^{4}-3 q^{2}+4-3 q^{-2}+q^{-4}\right) \\
\left(q-q^{-1}\right) P_{\mathcal{K}_{0}}= & a^{7}\left(q^{4}+q^{-4}\right)-a^{5}\left(q^{6}+1+q^{-6}\right)+a^{3}\left(q^{2}-1+q^{-2}\right) \\
(2.10)\left(q-q^{-1}\right) P_{\mathcal{K}_{0-}=} & a^{5}\left(q^{4}-q^{2}+2-q^{-2}+q^{-4}\right) \\
& +a^{3}\left(-q^{6}+q^{4}-3 q^{2}+3-3 q^{-2}+q^{-4}-q^{-6}\right) \\
& +a\left(q^{4}-2 q^{2}+3-2 q^{-2}+q^{-4}\right) .
\end{aligned}
$$

In the following, we investigate the HOMFLY homology $\bar{H}\left(\mathcal{K}^{\star}\right)$ in a similar way to Rasmussen's computation of $\bar{H}\left(9_{42}\right)$; see [15, Section 7.5].

Fix a sufficiently large $N \gg 1$. Recall that $\overline{5_{2}}$ and $\mathcal{K}_{0-}$ are KR-thin. Thus we can explicitly compute $\bar{H}_{N}\left(\overline{5_{2}}\right), \bar{H}_{N}\left(\overline{5_{2}}\right) \otimes M$ and $\overline{\bar{H}}_{N}\left(\mathcal{K}_{0-}\right)$, from (2.8), (2.9), (2.10) and their signatures with the aid of (2.3). Figure 5 is a superimposed diagram of generators of these homologies. Squares $(\square)$ represent generators of $\bar{H}\left(\overline{5_{2}}\right)$, solid 
$\operatorname{dots}(\bullet)$ for $\bar{H}\left(\overline{5_{2}}\right) \otimes M$ and hollow dots (०) for $\overline{\bar{H}}\left(\mathcal{K}_{0-}\right)$. The $j$-gradings are shifted so that they correspond to the power of $a$ in the HOMFLYPT polynomial $P_{\mathcal{K}^{\star}}(a, q)$ of $\mathcal{K}^{\star}$.

Claim 2.2. The $\delta$-gradings of the generators are $\delta(\square)=\delta(\circ)=2$ and $\delta(\bullet)=4$.

Proof. Since $\overline{5_{2}}$ is KR-thin with $\sigma\left(\overline{5_{2}}\right)=2$ and the $\delta$-degree of the map $\bar{H}_{N}\left(\mathcal{K}^{\star}\right) \rightarrow$ $\bar{H}_{N}\left(\overline{5_{2}}\right)$ in (2.5) is 0 , the square generators have $\delta(\square)=\sigma\left(\overline{5_{2}}\right)-0=2$. Also, since both of the $\delta$-degrees of $\bar{H}_{N}\left(\overline{5_{2}}\right) \otimes M \rightarrow \overline{\bar{H}}_{N}\left(\mathcal{K}_{0}\right)$ in $(2.6)$ and $\overline{\bar{H}}_{N}\left(\mathcal{K}_{0}\right) \rightarrow \bar{H}_{N}\left(\mathcal{K}^{\star}\right)$ in (2.5) are 1 , the solid dots have $\delta(\bullet)=\sigma\left(\overline{5_{2}}\right)+1+1=4$.

Since $\mathcal{K}_{0-}$ is KR-thin with $\sigma\left(\mathcal{K}_{0-}\right)=1$ and the $\delta$-degree of $\overline{\bar{H}}_{N}\left(\mathcal{K}_{0}\right) \rightarrow \bar{H}_{N}\left(\mathcal{K}^{\star}\right)$ in (2.5) is 1 , the hollow dots have $\delta(\circ)=\sigma\left(\mathcal{K}_{0-}\right)+1=2$.

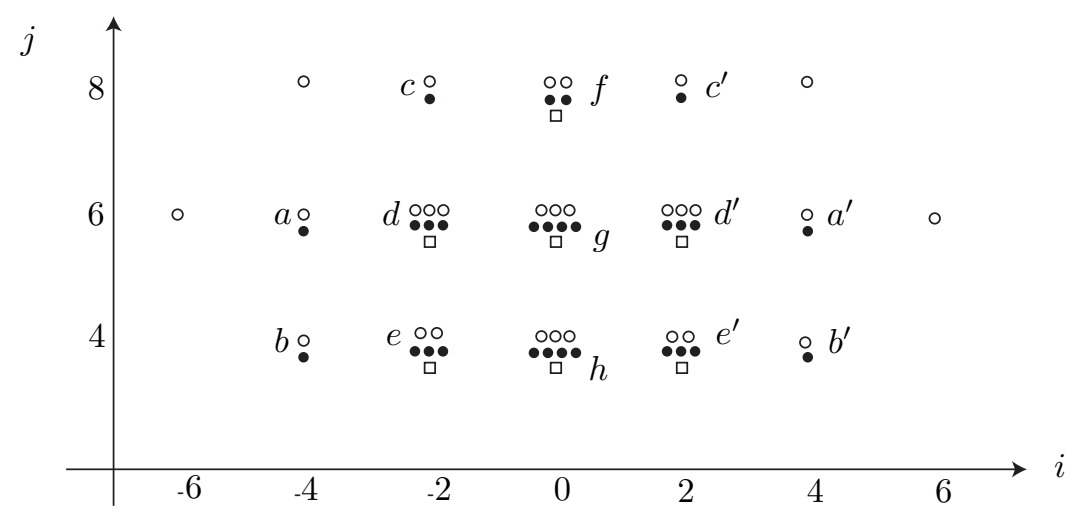

Figure 5. Possible generators of $\bar{H}\left(\mathcal{K}^{\star}\right)$. The $\delta$-gradings are $\delta(\circ)=\delta(\square)=2, \delta(\bullet)=4$.

Our $N$ is large enough so that non-trivial components of the map $\overline{\bar{H}}_{N}\left(\mathcal{K}_{0-}\right) \stackrel{(N, 1)}{\longrightarrow}$ $\bar{H}_{N}\left(\overline{5_{2}}\right) \otimes M$ preserve the lettered positions $a, b, \cdots, e^{\prime}$ of the generators corresponding to $\circ$ and $\bullet$ and the non-lettered hollow circle generators are the image of the map $\overline{\bar{H}}_{N}\left(\mathcal{K}_{0}\right) \stackrel{(-2 N, 0)}{\longrightarrow} \bar{H}_{N}\left(\mathcal{K}_{0-}\right)$. Noticing that square generators do not exist at the non-lettered positions, and since (2.5) is exact, we conclude that $\bar{H}_{N}\left(\mathcal{K}^{\star}\right)$ has generators at these non-lettered positions. Therefore, the maximal $j$-degree $\delta_{+}\left(\mathcal{K}^{\star}\right)$ of the HOMFLY-homology $\bar{H}\left(\mathcal{K}^{\star}\right)$ satisfies $\delta_{+}\left(\mathcal{K}^{\star}\right)=8$, which is equal to $w_{\mathcal{K}^{\star}}+b_{\mathcal{K}^{\star}}-1=6+3-1$.

We introduce the following claim, whose proof will be given shortly:

Claim 2.3. At position $b$ in Figure 5 , the group $\bar{H}\left(\mathcal{K}^{\star}\right)$ has rank 2.

Assuming Claim 2.3, the generators at $b$ attain $\delta_{-}\left(\mathcal{K}^{\star}\right)=4$, which is equal to $w_{\mathcal{K}^{\star}}-b_{\mathcal{K}^{\star}}+1=6-3+1$. Thus the KR-MFW inequality is sharp on $\mathcal{K}^{\star}$.

It remains to establish Claim 2.3.

Proof of Claim 2.3. Recall that when $N=2$ the reduced homology $\bar{H}_{2}$ is isomorphic to the reduced $s l(2)$ Khovanov-Rozansky homology [15, Proposition 3.12]. As 
stated in [10, Section 1], the $s l(2)$-homology is equivalent to the Khovanov homology. According to KhoHo by Shumakovitch [17, the Poincaré polynomial of the $\operatorname{sl}(2)$-homology $\bar{H}_{N=2}^{I, J}\left(\mathcal{K}^{\star}\right)$ is

(2.11)

$q^{4}+q^{4} t+q^{6} t^{2}+q^{8} t^{2}+q^{8} t^{3}+q^{10} t^{3}+2 q^{10} t^{4}+q^{12} t^{5}+q^{14} t^{5}+2 q^{14} t^{6}+q^{16} t^{7}+q^{18} t^{8}+q^{20} t^{9}$.

Recall the spectral sequence $E_{k}(2)$ which relates $\bar{H}^{i, j, k}\left(\mathcal{K}^{\star}\right)$ to $\bar{H}_{2}^{I, J}\left(\mathcal{K}^{\star}\right)$ [15, Theorem 2]. Position $b$ has $(i, j)=(-4,4)$. The generators corresponding to the term $q^{4}+q^{4} t$ of (2.11) have $q$-grading: $I=4$. Under the identification $I=i+N j=$ $i+2 j$ in (2.3), only position $b$, among the possible generators of $\bar{H}\left(\mathcal{K}^{\star}\right)$ plotted in Figure 5, meets $I=4$.

Remark 2.4. Since $\mathcal{K}_{k}=\Delta^{4 k} 12^{-6 k-1}$ and $\mathcal{L}_{k}=\Delta^{4 k+2} 12^{-6 k-1}$, where $\Delta=121$ is the positive half-twist, we notice that $\mathcal{L}_{k}=\Delta^{2} \mathcal{K}_{k}$. An analogous statement to [7, Corollary 1.7] of Kálmán is that the lower KR-MFW estimate for a braid $\beta$ is sharp if and only if the upper KR-MFW estimate is sharp for $\Delta^{2} \beta$. If this statement would hold, then Theorem 1.6 could imply the sharpness of the KR-MFW inequality for $\mathcal{L}_{1}$ and $\overline{\mathcal{L}}_{1}$.

\section{Proof of Theorem 1.7}

Lemma 3.1. Suppose that $D \in \mathcal{B}_{\mathcal{K}}$ is a closed braid diagram of $\mathcal{K}$ realizing the minimal braid index: $b_{D}=b_{\mathcal{K}}$. Focus on one site of $D$ and construct $D_{+}, D_{-}, D_{0}$ as specified in Figure 1 (one of the three must be D). Let $\alpha, \beta, \gamma \in\{+,-, 0\}$ be mutually distinct. Suppose $D_{\alpha}=D$.

If each of $D_{\beta}, D_{\gamma}$ can be positively destabilized $p>0$ times, then

$$
\left(w_{D}+b_{D}-1\right)-\delta_{+}(\mathcal{K}) \geq 2 p .
$$

If each of $D_{\beta}, D_{\gamma}$ can be negatively destabilized $n>0$ times, then

$$
\delta_{-}(\mathcal{K})-\left(w_{D}-b_{D}+1\right) \geq 2 n .
$$

Therefore, if $p+n>0$, then the $K R-M F W$ inequality is not sharp on $\mathcal{K}$.

Proof of Lemma 3.1. Suppose that $D=D_{+}, D_{\beta}=D_{-}$and $D_{\gamma}=D_{0}$. Similar arguments work for the other two cases: $D=D_{-}$or $D_{0}$.

Let $\mathcal{K}_{+}, \mathcal{K}_{-}, \mathcal{K}_{0}$ be the topological knot types of $D_{+}, D_{-}, D_{0}$ respectively. Maps $\bar{H}_{N}\left(\mathcal{K}_{-}\right) \stackrel{(N, 1)}{\longrightarrow} \bar{H}_{N}\left(\mathcal{K}_{0}\right) \stackrel{(N, 1)}{\longrightarrow} \bar{H}_{N}\left(\mathcal{K}_{+}\right)$in (2.4) raise the $q$-grading by $N$. Assuming that $N \gg 1$ (i.e., isomorphism (2.3) holds and $i+N j=I$ ) these maps raise the $j$-grading by 1 . So we shift the $j$-grading of $\mathcal{K}_{-}$(resp. $\left.\mathcal{K}_{0}\right)$ by 2 (resp. 1 ). Also by the exactness of (2.4) all the generators of $\bar{H}\left(\mathcal{K}_{+}\right)$come from those of $\bar{H}\left(\mathcal{K}_{-}\right), \bar{H}\left(\mathcal{K}_{0}\right)$. Therefore,

$$
\begin{aligned}
\min \left\{\delta_{-}\left(\mathcal{K}_{-}\right)+2, \delta_{-}\left(\mathcal{K}_{0}\right)+1\right\} & \leq \delta_{-}\left(\mathcal{K}_{+}\right) \\
\leq \delta_{+}\left(\mathcal{K}_{+}\right) & \leq \max \left\{\delta_{+}\left(\mathcal{K}_{-}\right)+2, \delta_{+}\left(\mathcal{K}_{0}\right)+1\right\}
\end{aligned}
$$

Let $\tilde{D}_{-}\left(\right.$resp. $\left.\tilde{D}_{0}\right)$ be a closed braid diagram obtained by applying positive destabilization $p$ times to $D_{-}$(resp. $\left.D_{0}\right)$. By the KR-MFW inequality, we have:

$$
\begin{aligned}
\delta_{+}\left(\mathcal{K}_{-}\right)+2 & \leq\left(w_{\tilde{D}_{-}}+b_{\tilde{D}_{-}}-1\right)+2=\left\{\left(w_{D_{-}}+b_{D_{-}}-1\right)-2 p\right\}+2 \\
& =\left(w_{D_{+}}-2\right)+b_{D_{+}}-1-2 p+2=\left(w_{D_{+}}+b_{D_{+}}-1\right)-2 p \\
\delta_{+}\left(\mathcal{K}_{0}\right)+1 & \leq\left(w_{\tilde{D}_{0}}+b_{\tilde{D}_{0}}-1\right)+1=\left(w_{D_{0}}+b_{D_{0}}-1-2 p\right)+1 \\
& =\left(w_{D_{+}}-1\right)+b_{D_{+}}-1-2 p+1=\left(w_{D_{+}}+b_{D_{+}}-1\right)-2 p .
\end{aligned}
$$


Combining them with (3.3) we obtain

$$
\delta_{+}\left(\mathcal{K}_{+}\right) \leq \max \left\{\delta_{+}\left(\mathcal{K}_{-}\right)+2, \quad \delta_{+}\left(\mathcal{K}_{0}\right)+1\right\} \leq\left(w_{D_{+}}+b_{D_{+}}-1\right)-2 p,
$$

which proves (3.1). Similarly, inequality (3.2) holds.

Proof of Theorem 1.7. Define braid diagrams $M_{+}, M_{-}, M_{0}$ differing at the shaded crossing in Figure 2,

$$
\begin{aligned}
& M_{-}=2^{x} 3^{y} \overline{1} \overline{2} 2^{z} 1^{w} 232 \overline{1} \quad=B M_{x, y, z, w}, \text { (the right sketch of Figure 2), } \\
& M_{+}=2^{x} 3^{y} \overline{1} \overline{2} 2^{z} 1^{w} 2321 \quad \stackrel{+}{\Longrightarrow} \tilde{M}_{+}=1^{x} 2^{y+1} 1^{2} 2^{z+1} 1^{w} 2, \\
& M_{0}=2^{x} 3^{y} \overline{1} \overline{2} 2^{z} 1^{w} 232 \quad \Longrightarrow \tilde{M}_{0}=2^{y} 1^{z+1} 2^{x+1} 1^{w+1} 2 .
\end{aligned}
$$

Here $\stackrel{+}{\Longrightarrow}$ means a positive destabilization and braid isotopy whose intermediate steps are shown in [8, Figure 5]. Note that $b_{\tilde{M}_{+}}=b_{\tilde{M}_{0}}=3$. On the other hand, [8. Theorem 2.8] proves that there exist infinitely many $(x, y, z, w)$ 's such that the braid index of knot type $B M_{x, y, z, w}$ is 4, i.e., the condition $b_{D}=b_{\mathcal{K}}$ of Lemma 3.1 is satisfied. Applying Lemma 3.1 we conclude that the KR-MFW inequality is not sharp on infinitely many $B M_{x, y, z, w}$ 's.

Remark 3.2. Although knot $9_{42}=B M_{-1,1,-2,-1}$ is realized by the BM-diagram, thus covered by Theorem 1.7. Rasmussen [15, Figure 14] has explicitly computed its reduced KR-homology $\bar{H}\left(9_{42}\right)$, which exhibits non-sharpness of the KR-MFW inequality on $9_{42}$.

\section{Maximal Bennequin numbers of $\mathcal{K}_{k}$ And $\mathcal{L}_{k}$}

The next lemma for Theorem 1.11 is due to Elrifai [4].

Lemma 4.1. [4, Proposition 2.2.8] The HOMFLYPT polynomial of $\mathcal{K}_{k}$ (resp. $\left.\mathcal{L}_{k}\right)$ coincides with the one for the $(2,6 k+1)$-torus knot $T_{2,6 k+1}$ (resp. $\left.T_{2,6 k+5}\right)$ :

$$
P_{\mathcal{K}_{k}}(a, q)=P_{T_{2,6 k+1}}(a, q), \quad P_{\mathcal{L}_{k}}(a, q)=P_{T_{2,6 k+5}}(a, q) .
$$

Proof of Theorem 1.11. By Theorem 1.5] and Proposition 1.10, it follows that Conjectures 1.8 and 1.9 hold for the class $B_{3}^{\prime}$.

Noticing that the braid words $(21 \overline{2})$ and $(\overline{2} 12)$ are quasipositive factors, $\mathcal{K}_{k}, \mathcal{L}_{k}$ have quasipositive braid representations:

$$
D_{\mathcal{K}_{k}}=\overline{2}\left(\begin{array}{llllll}
1 & 2 & 2 & 1 & \overline{2}
\end{array}\right)^{2 k} 1, \quad D_{\mathcal{L}_{k}}=\left(\begin{array}{llllll}
1 & 2 & 2 & 1 & \overline{2}
\end{array}\right)^{2 k-1}\left(\begin{array}{llll}
1 & 2 & 2 & 1
\end{array}\right)^{2} 1 .
$$

Rudolph's slice-Bennequin inequality [16] is that for any $D \in \mathcal{B}_{\mathcal{K}}$ we have $2 g_{4}(\mathcal{K}) \geq w_{D}-b_{D}+1=\beta_{D}+1$. Moreover, if $D \in \mathcal{B}_{\mathcal{K}}$ is quasipositive, then equality holds.

Therefore, the above quasipositive diagrams $D_{\mathcal{K}_{k}}, D_{\mathcal{L}_{k}}$ attain the maximal Bennequin numbers. Since $b_{\mathcal{K}_{k}}=b_{D_{\mathcal{K}_{k}}}=3$ and $b_{\mathcal{L}_{k}}=b_{D_{\mathcal{L}_{k}}}=3$ (the minimal braid index), Conjecture 1.9 holds for $\mathcal{K}_{k}$ and $\mathcal{L}_{k}$.

By Lemma 4.1 and the sharpness of the MFW-inequality on torus links [5, Corollary 2.4], we can compute the minimal and the maximal $a$-degrees of $P_{\mathcal{K}_{k}}(a, q)$ and $P_{\mathcal{L}_{k}}(a, q)$ :

$$
d_{-}\left(\mathcal{K}_{k}\right)=6 k, \quad d_{+}\left(\mathcal{K}_{k}\right)=6 k+2, \quad d_{-}\left(\mathcal{L}_{k}\right)=6 k+4, \quad d_{+}\left(\mathcal{L}_{k}\right)=6 k+6 .
$$

Since $w_{D_{\mathcal{K}_{k}}}+b_{D_{\mathcal{K}_{k}}}-1=6 k+2$, the right side of the MFW inequality is sharp on $\mathcal{K}_{k}$, i.e., $d_{+}\left(\mathcal{K}_{k}\right)=w_{D}+b_{D}-1$. This means that on its mirror image $\overline{\mathcal{K}}_{k}$, the 
left side of the MFW inequality is sharp: $w_{\bar{D}}-b_{\bar{D}}+1=d_{-}\left(\overline{\mathcal{K}}_{k}\right)$, where $\bar{D}$ is the mirror image of $D_{\mathcal{K}_{k}}$. Thus both Conjectures 1.8 and 1.9 hold for $\mathcal{K}_{k}$ and $\overline{\mathcal{K}}_{k}$.

We remark that since $w_{D_{\mathcal{L}_{k}}}+b_{D_{\mathcal{L}_{k}}}-1=6 k+8>6 k+6=d_{+}\left(\mathcal{L}_{k}\right)$ (the MFW inequality is not sharp on $\mathcal{L}_{k}$ ), the same argument does not apply to $\mathcal{L}_{k}$.

Remark 4.2. After completion of this paper, the author was informed that Conjecture 1.8 for 3-braids was proved by Stoimenow [18, Theorem 7.1].

\section{ACKNOWLEDGMENTS}

The author would like to thank the referee and the editor for patiently reading this paper and for giving many valuable suggestions, comments and corrections. Conversations with Joan Birman, Michel Boileau, John Etnyre, Nathan Ryder, Alexander Stoimenow and Hao $\mathrm{Wu}$ were very helpful in finishing this work. The author is especially grateful to Jacob Rasmussen for explaining the KR-homology and pointing out Claim 2.3.

\section{REFERENCES}

1. Bennequin, D., Entrelacements et équations de Pfaff, Astérisque 107-108 (1983), 87-161. MR753131 (86e:58070)

2. Birman, J. S. and Menasco, W. W., Studying links via closed braids, III: Classifying links which are closed 3-braids, Pacific J. Math. 161 (1993), no. 1, 25-113. MR1237139(94i:57005)

3. Dunfield, N. M., Gukov, S. and Rasmussen, J., The superpolynomial for knot homologies, Experiment. Math. 15 (2006), no. 2, 129-159. MR.2253002 (2008c:57020)

4. Elrifai, E. A., Positive braids and Lorenz links, Ph.D. thesis, Liverpool University, 1988.

5. Franks, J. and Williams, R. F., Braids and the Jones polynomial, Trans. Amer. Math. Soc. 303 (1987), 97-108. MR896009 (88k:57006)

6. Jones, V. F. R., Hecke algebra representations of braid groups and link polynomials, Ann. of Math. (2) 126 (1987), 335-388. MR908150 (89c:46092)

7. Kálmán, T., Meridian twisting of closed braids and the HOMFLY polynomial, arXiv:0803.0103

8. Kawamuro, K., The algebraic crossing number and the braid index of knots and links, Algebraic \& Geometric Topology 6 (2006), 2313-2350. MR2286028 (2007k:57012)

9. Kawamuro, K., Conjectures on the braid index and the algebraic crossing number, Intelligence of Low Dimensional Topology 2006, Knots and Everything, 40, World Scientific Publ. Co., Hackensack, NJ, 2007, 151-155. MR2371720

10. Khovanov, M. and Rozansky, L., Matrix factorizations and link homology, Fund. Math. 199 (2008), no. 1, 1-91. MR2391017

11. Khovanov, M. and Rozansky, L., Matrix factorizations and link homology. II, Geom. Topol. 12 (2008), no. 3, 1387-1425.

12. Malešič, J. and Traczyk, P., Seifert circles, braid index and the algebraic crossing number, Topology Appl. 153 (2005), no. 2-3, 303-317. MR2175354 (2006i:57013)

13. Morton, H. R., Seifert circles and knot polynomials, Math. Proc. Cambridge Philos. Soc. 99 (1986), 107-109. MR809504 (87c:57006)

14. Murasugi, K., On the braid index of alternating links, Trans. Amer. Math. Soc. 326 (1991), no. 1, 237-260. MR 1000333 (91j:57009)

15. Rasmussen, J., Some differentials on Khovanov-Rozansky homology, math.GT/0607544

16. Rudolph, L., Quasipositivity as an obstruction to sliceness. Bull. Amer. Math. Soc. (N.S.) 29 (1993), no. 1, 51-59. MR1193540 (94d:57028)

17. Shumakovitch, A., KhoHo: a program for computing Khovanov homology, www.geometrie. $\mathrm{ch} / \mathrm{KhoHo} /$

18. Stoimenow, A., Properties of closed 3-braids, math/0606435 
19. Wu, H., Braids, transversal links and the Khovanov-Rozansky theory, Trans. Amer. Math. Soc. 360 (2008), no. 7, 3365-3389. MR2386230

20. Yamada, S., The minimal number of Seifert circles equals the braid index of a link, Invent. Math. 89 (1987), no. 2, 347-356. MR894383 (88f:57015)

Department of Mathematics, Rice University, Houston, Texas 77005

Current address: School of Mathematics, The Institute for Advanced Study, Princeton, New Jersey 08540

E-mail address: kk6@ias.edu 